\title{
Short Range Remote Powering of Implanted Electronics for Freely Moving Animals
}

\author{
Enver G. Kilinc ${ }^{\star}$, Mehrdad A. Ghanad ${ }^{\star}$, Franco Maloberti ${ }^{\diamond}$, and Catherine Dehollain ${ }^{\star}$ \\ * RF-IC Group, Ecole Polytechnique Fédérale de Lausanne, Lausanne, Switzerland \\ $\diamond$ Integrated Microsystem Laboratory, Universitá degli Studi di Pavia, Pavia, Italy \\ Email: enver.kilinc@epfl.ch, franco.maloberti@unipv.it, catherine.dehollain@epfl.ch
}

\begin{abstract}
An implantable system for monitoring vital parameters via bio-sensors inside freely moving laboratory animals and its powering system are presented. The required $2 \mathrm{~mW}$ are harvested by the magnetic coupling with an external coil placed under the living space of the animal. The servo $X-Y$ rails move the external coil and track the animal. Dynamic power-adaptation keeps the harvested power level constant against misalignments of transmitting coil and moving animal. Entire system and basic blocks integrated using a 0.18 um CMOS technology are presented. Experimental results show the effectiveness of the powering system.
\end{abstract}

\section{INTRODUCTION}

More and more electronic systems implanted in small animals for investigating new treatments need effective powering methods [1]. Especially, the small animals are used in research efforts for developing new drugs [2]. The requirement for the implanted system are typically small volume, data communication, and multiple sensing interfaces. Moreover, it is necessary to power the system externally because available batteries in the market do not allow a suitable autonomy.

Another important request is that the living conditions of animals must be natural so that the measured result are not biased. This means that the animal must be in a comfortable environment and capable to move freely [3]. However, delivering power to a freely moving animal is an important design challenge: the efficiency of the remote powering system must be high and almost independent on the animal position. These constraints motivate this research work that involves the design of a suitable powering system with an effective level of both transmitted power and data.

The requirement of harvesting an almost constant amount of power can be met by modulating the transmitted power, by using multiple power sources and activating the source which is closer to the target or moving the power source, as discussed here, by a servo tracking system. Moreover, the form of energy used for the transmission is important for achieving uniformity and efficiency.

\section{Choice of The Powering System}

There are several methods for remote powering. The power can be delivered via electro-magnetic waves, ultrasound, or magnetic field transmission. The choice depends on the tradeoffs between many parameters: the level of power needed, the distance between the power source and the target, the power transmission environment.
For electro-magnetic transmission power decreases as the square of the distance. A frequency range in between few hundred $\mathrm{MHz}$ to $\mathrm{GHz}$ is used to have a small antenna size. In order to have a received power of $2 \mathrm{~mW}$ according to the Friis transmission equation:

$$
P_{r}=P_{t} G_{t} G_{r}\left(\frac{\lambda}{4 \pi d}\right)^{2}
$$

where $P_{r}, P_{t}, G_{t}, G_{r}, \lambda$, and $d$ present the received power level, the transmitted power level, the transmitter antenna gain, the receiver antenna gain, the wavelength of the electromagnetic wave and the distance between the two antennas, respectively. It is necessary to have $P_{t}$ more than $1 \mathrm{~W}$ for $12 \mathrm{~cm}$ distance at $2.42 \mathrm{GHz}$ [4]. The value is close to Specific Absorption Rate (SAR) limit which is defined by regulations.

Ultrasound is also a solution for wireless power transmission for deeply implanted systems. The equation 2 defines the received power level for ultrasound [5].

$$
P_{r}=\eta_{A E} I_{A C} S_{A}
$$

where $P_{r}, I_{A C}, S_{A}$ represent the received power from the ultrasound link, acoustic intensity and surface area of the transducer, respectively. Additionally, $\eta_{A E}$ defines the acoustoelectric efficiency. According to the study in [5], the power transmission efficiency is very good in water. In order to obtain $P_{r}=2 \mathrm{~mW}, 20 \mathrm{~mW}$ of source power is enough at $11 \mathrm{~cm}$ distance. Unfortunately, $\eta_{A E}$ is very low in the air and this would require to transmit a power level that is perceived by the animal. This power level disturbs the comfort of the animal.

The received power on a magnetically coupled coils is defined in [6];

$$
P_{r}=P_{t} \frac{\mu_{0}^{2} \pi^{2} N_{t}^{2} N_{r}^{2} r_{t}^{4} r_{r}^{4} \omega^{2}}{16 R_{t} R_{r}\left(r_{t}^{2}+d^{2}\right)^{3}}
$$

where $\mu_{0}$ is the permeability of free space. $P_{r}$, and $P_{t}$ are the received and the transmitted power levels. $N_{i}, r_{i}, R_{i}$ represent the number of turns of the coils, the radius of the coils, the resistance of the coils, respectively. $d$ is distance between the coils. For the same received power $(2 \mathrm{~mW})$, $20 \mathrm{~mW}$ transmitted power is needed at $10 \mathrm{~cm}$ distance for magnetically coupled coils given in [6]. The studies in [7], and [8] also show that the optimal choice is using magnetic field transmission for short-range remotely powered systems. 


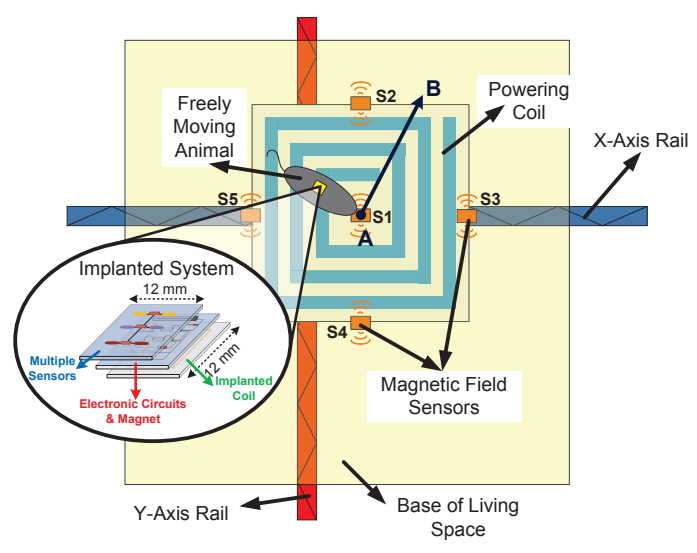

Fig. 1. Overall view of entire system

Even for this method there are possible problems associated to the need of generating an almost uniform magnetic signal in the area of freely moving animal. There are two possible solutions. First, to use an array of coils, possibly acting by detecting the position of the animal [9] or to use a single coil whose position is servo-controlled. The study of the two solution leads to the choice of the servo-controlled method because it ensures an optimal uniform magnetic power.

\section{SYSTEM DESCRIPTION}

Fig. 1 illustrates the overall view of the entire system. It consists of an external unit and implanted unit. The external unit has the powering coil which is controlled by servocontrolled rails to deliver optimum power to the implanted unit. In addition, some commands are transmitted to setup the sensors and the data is received by the external unit. The implanted unit is a remotely powered multi-sensor system. The unit has a 3-layer platform as shown in Fig. 1. On the top, the sensors are placed to have a contact with the body. The sensors are used to measure the glucose and the drug level of the vicinity of the implanted system. Additionally, $\mathrm{pH}$ and temperature of the same area is also needed to calibrate the sensors. In order to activate the sensors and transmit the data, electronic circuits are located in the middle layer. In addition, a permanent magnet is also added to detect the place of the implanted unit. In the bottom, the implanted coil is placed to generate a supply from the available magnetic field. Fig. 2 shows the block diagram of both units.

\section{A. Power Transmission}

The power is transferred over an optimized inductive link at $13.56 \mathrm{MHz}$ which is specialized for inductive application band. The power activates batteryless implanted system and measures vital parameters in the body. The sizes of the powering and the implanted coils are $80 \times 80 \mathrm{~mm}$ and $12 \times 12 \mathrm{~mm}$ respectively. The minimum distance between the powering coil and the implanted coil is $30 \mathrm{~mm}$ when two coils are concentric.

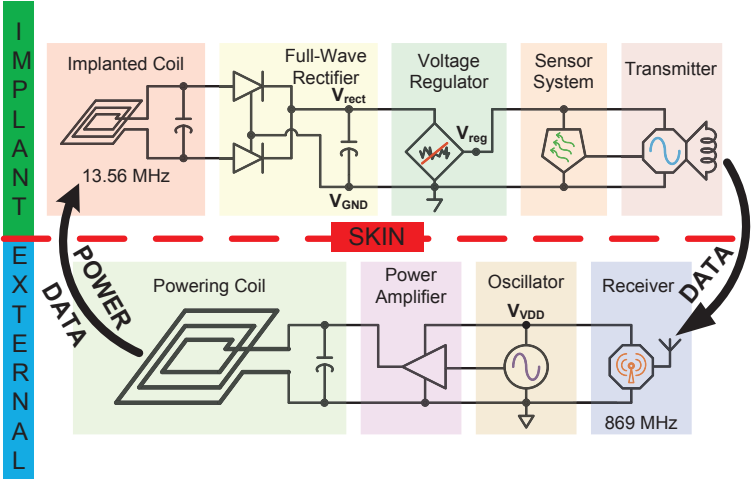

Fig. 2. Block diagram of implanted and external units

\section{B. Data Communication}

The data collected from sensors are transmitted by an integrated transmitter to the external unit at $869 \mathrm{MHz}$. This band is an ISM band for data communications. This high frequency band is chosen for the high data rate communication and not to create interference between the powering and communication channels. In addition, the data is received by a custom designed receiver at the external side. In order to calibrate the sensors and establish the measurement, some commands are transmitted together with power at the cost of a limited reduction of power efficiency [10]. The commands are received by an Amplitude Shift Keying (ASK) demodulator.

\section{BUILDING BLOCKS}

The system described in the previous section includes some relevant building blocks. They are described in more details in the following section.

\section{A. Magnetic Power Transmitter}

Fig. 3 shows the simple scheme used for the power transmission. The power is transferred via magnetically coupled coils $L_{2}$, and $L_{3}$. The capacitors $C_{2}$, and $C_{3}$ are also used to tune the coils to same operation frequency $(13.56 \mathrm{MHz})$ to increase the power efficiency. The scheme includes a sine wave generator followed by a class-E power amplifier. $L_{1}$ is a RF-choke inductor to bias the transistor and $C_{1}$ is a shuntcapacitor to sustain the current for the inductive link. The values of $L_{1}$ and $C_{1}$ are optimized for maximizing power transmission efficiency. The sine wave signal is amplified by this amplifier and the magnetic field is generated on the powering coil.

The supply voltage of the amplifier $\left(V_{\text {sup }}\right)$ is controlled by a power feedback control. When the power at the implanted unit is insufficient, the Power Feedback Data (PFD) is transmitted as "0" which increases the $V_{\text {sup }}$ voltage by charging the $C_{\text {sup }}$ capacitor and as well as the transmitted power level. On the contrary, if the power level at the implanted unit is high, the PFD is transmitted as " 1 " and the $V_{\text {sup }}$ voltage decreases. 


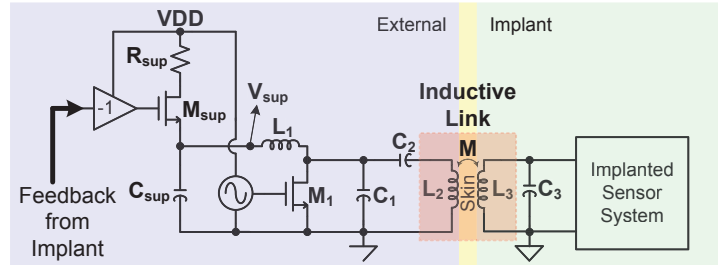

Fig. 3. Simple scheme of power transmission with dynamic power adaptation

\section{B. Magnetic Power Receiver}

This block [11] is made by an implanted coil, a full-wave rectifier, and a voltage regulator as illustrated in Fig. 2. The generated magnetic field induces the current on the implanted coil. The passive full-wave rectifier charges a storage capacitor which is also used to provide supply voltage $\left(V_{\text {rect }}\right)$ to the implanted system when the wireless power transmission is interrupted. The high-speed voltage regulator suppresses the ripples on $V_{\text {rect }}$ voltage and generates a clean and stable supply voltage $\left(V_{r e g}\right)$ for the sensor system. The power level at the implanted unit is controlled by following $V_{\text {rect }}$ voltage and it is compared to a reference voltage and the PFD is transmitted to the external unit to adjust $V_{\text {sup }}$ voltage of the amplifier.

\section{Data Transmitters}

The measurement data is transmitted with on-off keying (OOK) modulated signals by an integrated low-power transmitter as shown in Fig. 2. The transmitter is made by a freerunning LC oscillator which is composed of two NMOS and two PMOS cross-coupled transistors. The external inductance of LC tank is also used as a transmitter antenna which allows short-range communication without using extra components. The commands are transmitted from the external unit to the implanted unit by modulating the amplitude of the powering signal.

\section{Data Receivers}

In order to reduce the overall power consumption of the implanted unit the data is transferred by a low-power transmitter. However, the free-running oscillator has a large frequency deviation. Therefore, a custom design receiver is needed. The receiver is designed in order to operate under the frequency shift of the transmitter. The commands are received by an integrated ASK demodulator in the implanted unit.

\section{E. Servo Control of External Powering Coil}

The power transmission efficiency changes drastically when the implanted coil moves from the center to the edges of the powering coil. Moreover, the power transmission stops when the implanted coil stays on the edge of the powering coil. Therefore, an Intelligent Remote Powering (IR-Power) system is needed to always deliver the power efficiently [12]. Fig. 4 shows the implementation of the IR-Power system for freely moving animals.

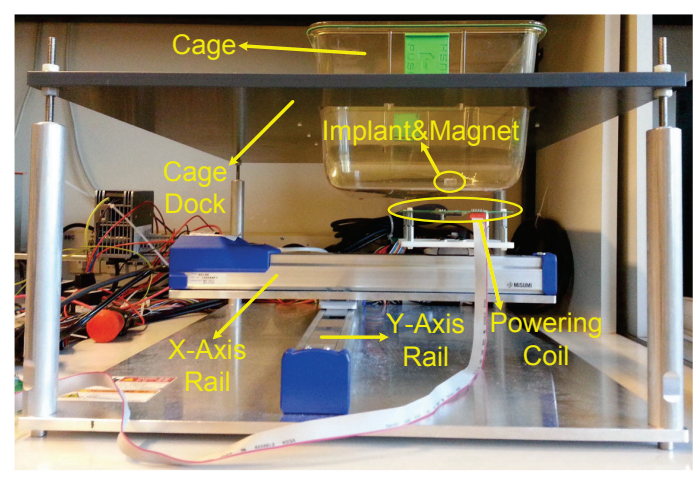

Fig. 4. Implementation of IR-Power system for freely moving animals

The IR-Power system has $\mathrm{X}$ and $\mathrm{Y}$ rails and the powering coil is moved on the $\mathrm{X}$ and $\mathrm{Y}$ axes by using two rails. The position of the animals is detected by a static magnetic field of a permanent magnet implanted in the animal. A suitable control algorithm determines the driving signal of the $\mathrm{X}$ and $\mathrm{Y}$ rails. The key of the algorithm is to ensure a high alignment accuracy of $1.5 \mathrm{~cm}$ as verified by the experimental results presented in next section.

The permanent magnet is detected by the magnetic field sensors as illustrated in Fig. 1. The output voltage of magnetic field sensor changes according to the available static magnetic field strength. Assuming that the animal moves from point $A$ to point $B$. When the animal is at the point $A$, the sensor $S 1$ which is located in the center of the powering coil has the maximum output voltage compared to the other sensors on the edges of the powering coil. However, the output of the sensor $S 1$ starts to decrease when the animal moves from the point $A$ to the point $B$. Additionally, the outputs of the sensors $S 2$ and $S 3$ increase correspondingly. Accordingly, the IR-Power system moves the powering coil to north-east direction until the output of the sensor $S 1$ is again maximized. Therefore, the remote powering is achieved efficiently without any interruption.

\section{Measurement Results}

The testbench of IR-Power system shown in Fig. 4 emulates the real environment. The measurement of the power transmission efficiency of the inductive link leads to the result of Fig. 5. The variable $d$ is the distance between the centers of the two coils. $h$ is the gap between powering coil and implanted coil. $h$ accounts for the gap between moving coil, the wooded floor of the living space, and the height of the implanted part in the animal $(2 \mathrm{~mm})$. Its value is $3 \mathrm{~cm}$. In addition, $m$ is the lateral misalignment of the implanted coil according to center of the powering coil. The powering coil size is $8 \times 8 \mathrm{~cm}$ and $d$ changes from $3 \mathrm{~cm}$ to $5 \mathrm{~cm}$ (when the implanted coil is on the edge of the powering coil). The power transmission efficiency of the inductive link decreases with distance and goes almost to zero when $d=5 \mathrm{~cm}$. Indeed, the IR-Power system, which tracks the freely moving animal in the living space, has some delay to place the powering coil under the animal. Although, the maximum speed of the rails is higher than the animal, the 


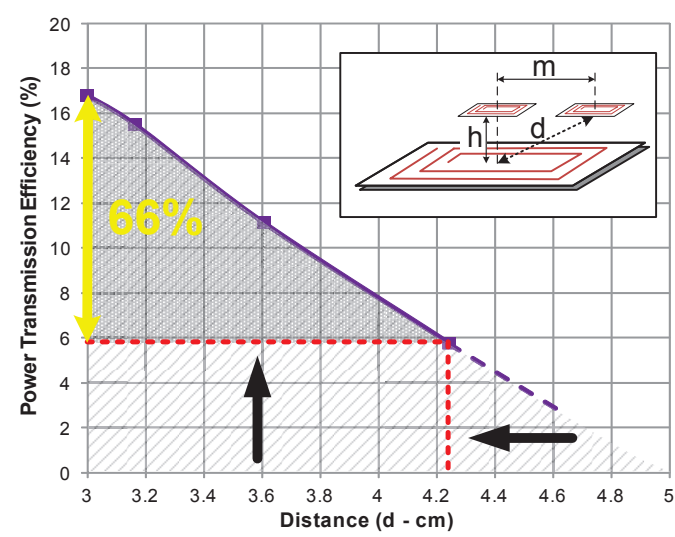

Fig. 5. Power transmission efficiency vs. distance

IR-Power system has a delay to activate the mechanical part. The delay gives rise to a maximum distance of $d=4.2 \mathrm{~cm}$. Moreover, the measurement quantifies the required range of dynamic power-adaptation. It results from the accuracy of coil alignment that, in the worst case leads to a maximum value of $d=4.2 \mathrm{~cm}$. For that value the harvested power drops by $66 \%$ with respect to the perfect coil alignment. Since the power transmission efficiency drops from $16.89 \%$ to $5.7 \%$, the dynamic power-adaptation must response accordingly to keep the harvested power at the required $2 \mathrm{~mW}$ level.

Fig. 6 shows experimental results of dynamic poweradaptation technique. The dynamic power-adaptation is tested by changing the coupling between the powering and the implanted coils. When the coupling between the coils is increased, $V_{\text {rect }}$ voltage also increases. After $V_{\text {rect }}$ exceeds $2.31 \mathrm{~V}$, the comparator output changes to "1" as shown in Fig. 6. The PFD = 1 means that the power in the implanted unit is high, hence $V_{\text {sup }}$ voltage reduces. On the contrary, the comparator output gives to "0" when $V_{\text {rect }}$ drops under $2.23 \mathrm{~V}$ and results with increase on $V_{\text {sup }}$ voltage. $V_{\text {rect }}$ voltage has a hysteresis loop and the average is $2.27 \mathrm{~V}$. Moreover, $V_{\text {reg }}$ is regulated supply voltage of the sensor system and has an average value of $1.808 \mathrm{~V}$. The PFD is transmitted by the integrated transmitter to the custom designed receiver to adjust $V_{\text {sup }}$ voltage according to the implanted unit request as illustrated in Fig. 6. Accordingly, the power drop due to the IR-Power system is also compensated by the dynamic poweradaptation and the required power is received constantly.

\section{CONCLUSION}

This paper presents a batteryless remotely-powered implantable multi-sensor system and servo-controlled remote powering system for freely moving laboratory animals. The implanted system demands continuous $2 \mathrm{~mW}$ of received power via the optimized inductive link. The powering coil is moved with a suitable control algorithm by the IR-Power system under the living space of the animal. Additionally, the IR-Power system tracks the animal movements and records the data. The harvested power level in the implanted system is kept constant by the dynamic power-adaptation against

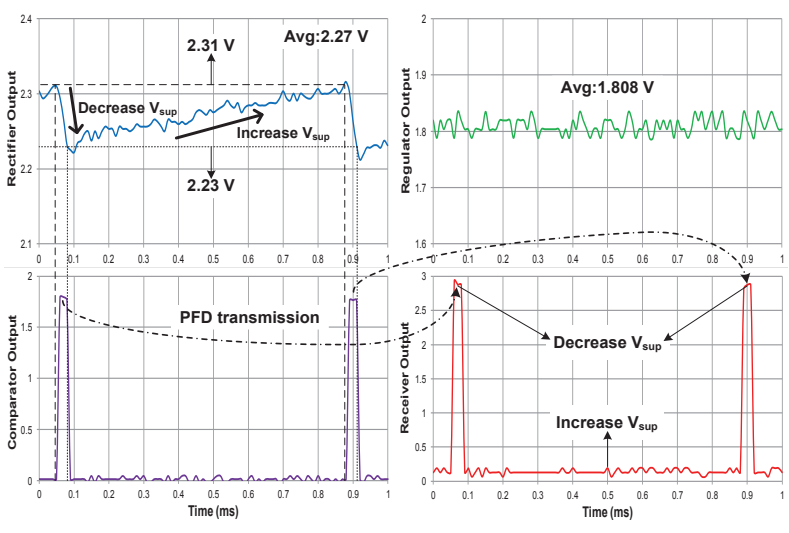

Fig. 6. Dynamic power-adaptation technique for freely moving animal

misalignments of the powering coil and the freely moving animal. The entire system and main blocks are fabricated in 0.18 um CMOS technology. The experimental results show that $V_{\text {rect }}$ voltage varies between $2.23 \mathrm{~V}$ and $2.31 \mathrm{~V}$. Moreover, the average of $V_{\text {reg }}$ voltage is $1.808 \mathrm{~V}$.

\section{ACKNOWLEDGMENT}

The authors thank to G. Conus, F. Mazzilli, G. Yilmaz, O. Atasoy and B. Kawkabani for their valuable feedback and help. Also, the authors are grateful to X. Warot and R. Doenlen from Life Science department, EPFL for their supports on rodent animals. This project is supported by Swiss National Funding (SNF) through Sinergia and PlaCiTUS Initiatives.

\section{REFERENCES}

[1] D. J. Young, "An RF-powered wireless multi-channel implantable biosensing microsystem", in Proc. IEEE Conf. Eng. Med. Biol. Soc., 2010, pp. 6413-6416.

[2] V. Marrella, et. al., "Anti-CD3 $\epsilon$ mAb improves thymic architecture and prevents autoimmune manifestations in a mouse model of Omenn syndrome: therapeutic implications," in Blood, 2012, vol. 120, no. 5, pp. 1005-1014.

[3] Q. Wang, H.R. Brunner, M. Burnier, "Determination of cardiac contractility in awake unsedated mice with a fluid-filled catheter," in Am. J. Physiol. Heart Circ. Physiol., 2004, vol. 286, no. 2, pp. H806-14.

[4] O. Kazanc, et. al., "Simulation oriented rectenna design methodology for remote powering of wireless sensor systems," in Proc. IEEE ISCAS 2012, 2012, pp. 2877-2880.

[5] F. Mazzilli, et. al., "Ultrasound energy harvesting system for deep implanted-medical-devices (IMDs)," in Proc. IEEE ISCAS 2012, 2012, pp. 2865-2868.

[6] D.C. Yates, A.S. Holmes, A.J. Burdett, "Optimal transmission frequency for ultralow-power short-range radio links," in IEEE Trans. Circuits Syst. I, 2004, vol. 51, no. 7, pp. 1405-1413.

[7] A. Denisov, E. Yeatman, "Ultrasonic vs. inductive power delivery for miniature biomedical implants," in Body Sensor Networks (BSN), 2010 International Conference on, 2010, pp. 8489.

[8] K. Finkenzeller, RFID Handbook, John Wiley \& Sons, 2003.

[9] E.G. Kilinc, et. al., "Intelligent cage for remotely powered freely moving animal telemetry systems," in Proc. IEEE ISCAS 2012, 2012, pp. 22072210.

[10] E.G. Kilinc, et. al., "FoM to compare the effect of ASK based communications on remotely powered systems," in Proc. PRIME'11 Conf., pp.29-32, 2011.

[11] E.G. Kilinc, et. al., "Remotely powered telemetry system with dynamic power-adaptation for freely moving animals," in IEEE BioCAS 2012, 2012, pp. 260-263.

[12] E.G. Kilinc, C. Dehollain, "Intelligent Remote Powering," EPO Patent 12180919.8, August 17, 2012. 